\title{
Differences in carcinoembryonic antigen levels between colon and rectal cancer
}

\author{
YUNLONG DING ${ }^{1 *}$, WEIBO XUAN ${ }^{2 *}$, CHUNLIN CHEN $^{2 *}$, ZHE CHEN $^{1}$, \\ ZIYI YANG ${ }^{2}$, YUNFEI ZUO ${ }^{2}$ and SHUANGYI REN ${ }^{1}$ \\ ${ }^{1}$ Department of General Surgery, The Second Affiliated Hospital of Dalian Medical University, Dalian, Liaoning 116023; \\ ${ }^{2}$ Department of Clinical Biochemistry, Dalian Medical University, Dalian, Liaoning 116044, P.R. China
}

Received February 2, 2014; Accepted April 16, 2014

DOI: $10.3892 / \mathrm{mco} .2014 .286$

\begin{abstract}
The aim of the present study was to investigate the levels of the serum tumor biomarker carcinoembryonic antigen (CEA) in patients with carcinoma of the colon and rectum in different clinical stages. Colorectal cancer (CRC) is one of the most commonly diagnosed types of cancer worldwide and previous studies have reported rapidly updated therapeutic regimes. While the majority of studies focus on CRC as a single entity, certain studies distinguish colon cancer (CC) from rectal cancer (RC), as there is a hypothesis stating that $\mathrm{CC}$ and $\mathrm{RC}$ are two naturally different entities. CEA is reported to be an important tumor-associated antigen overexpressed in $\mathrm{CRC}$, which is routinely detected as a significant indicator of CRC. Our study aimed to identify potential differences in the expression of CEA between $\mathrm{CC}$ and $\mathrm{RC}$, which may, to some degree, reflect the natural differences between the two. We investigated 240 CRC cases between July, 2010 and December, 2012 from The First and Second Affiliated Hospitals of Dalian Medical University, including 117 CC and 123 RC patients with tumors classified by Duke's staging as A-D. The serum CEA level was measured preoperatively by radioimmunoassays as a routinely used auxiliary indicator. The expression of CEA differed between $\mathrm{CC}$ and $\mathrm{RC}$, with the former exhibiting variation among the four stages, whereas no variation was observed in RC. In addition, there were differences between $\mathrm{CC}$ and $\mathrm{RC}$ regarding the CEA level in stage $\mathrm{C}$ and $\mathrm{D}$. Furthermore, the CEA level in stage $\mathrm{C}$ of CC was significantly lower compared
\end{abstract}

Correspondence to: Professor Shuangyi Ren, Department of General Surgery, The Second Affiliated Hospital of Dalian Medical University, 467 Zhongshan Road, Dalian, Liaoning 116023, P.R. China E-mail: rsydl@aliyun.com; rsydl@yahoo.com.cn

Professor Yunfei Zuo, Department of Clinical Biochemistry, Dalian Medical University, 9 Lushunnan Road Xiduan, Dalian, Liaoning 116044, P.R. China

E-mail: zyf04112002@aliyun.com; zyf04112002@yahoo.com.cn

*Contributed equally

Key words: colorectal cancer, carcinoembryonic antigen, colon and rectal cancer, different diseases, stage $\mathrm{C}$ to that in any other stage. In conclusion, the intrinsic distribution of the CEA level between CC and RC suggests that CC and $\mathrm{RC}$ may be two naturally different entities; the significantly low CEA level in stage $\mathrm{C}$ of $\mathrm{CC}$ indicates that stage $\mathrm{C}$ may be crucial in the evolution of $\mathrm{CC}$.

\section{Introduction}

Colorectal cancer (CRC) is the second most commonly diagnosed type of cancer in females and the third in males worldwide, with an estimated $>1.2$ million new cancer cases and 608,700 deaths in 2008 (1). While colon cancer (CC) and rectal cancer (RC) are often referred to as CRC due to their adjacent anatomic location, several studies investigating risk factors to underlying molecular mechanisms, have reported differences between CC and RC.

Dietary risk factors appeared to be considerably different between CC and RC (2) and the Cancer Genome Atlas Network reported differences between tumors located in the right colon and all other sites (3). Whether CC and RC are of a markedly different nature has not been clearly determined and the number of clinical studies that have emphasized the differences between $\mathrm{CC}$ and $\mathrm{RC}$ is currently limited.

Several tumor markers, including carcinoembryonic antigen (CEA), carbohydrate antigen (CA) 19.9 and CA 12.5, have been detected in a number of malignancies, including CRC. The levels of CEA and CA19.9 are often elevated in advanced CRC (4) and have been considered as an early sign of CRC recurrence (5).

CEA, originally identified in human fetal intestine and adult CC tissue in 1965 by Gold and Freedman (6), was subsequently characterized as a glycosylated cell-surface glycoprotein with a molecular weight of 180,000 Da (7); further research identified it as a significant tumor-associated antigen that is highly overexpressed in breast, lung and pancreatic cancer and, particularly, in CRC $(8,9)$. CEA is routinely detected as a tumor biomarker and an auxiliary indicator for the preoperative diagnosis of CRC (10), as well as an early predictor of recurrence (5), which to some extent accounts for CEA reflecting the nature of $\mathrm{CRC}$.

As there are currently few available studies reporting the variations in the expression of CEA between $\mathrm{CC}$ and $\mathrm{RC}$, the present study aimed to investigate the possible differences in 
the serum CEA level between CC and RC, which may highlight the distinct natures of these two types of cancer.

\section{Materials and methods}

Serum CEA concentrations. A total of 240 cases were investigated between July, 2010 and July, 2012 from The First and Second Affiliated Hospitals of Dalian Medical University, including $117 \mathrm{CC}$ and $123 \mathrm{RC}$ patients. The patients underwent potentially curative resection of colorectal carcinomas, which were classified as Duke's stage A-C and patients diagnosed as stage $\mathrm{D}$ were classified by biopsy or radiographic findings (endorectal ultrasound, computed tomography and magnetic resonance imaging). The disease stage was determined according to the Dukes' staging system, which is equivalent to the TNM staging system of the American Joint Committee on Cancer (11). All the clinical findings were prospectively recorded in detail and stored in computerized files, which included name, gender, age, family history, CEA level and the location and stage of the tumors.

The serum CEA concentrations were measured preoperatively in the 240 patients by radioimmunoassays performed in the clinical laboratories of the two affiliated hospitals. CEA kits from Roche Diagnostics (Basel, Switzerland) were used for detection following the manufacturer's instructions. High CEA concentrations were redefined as those $>5 \mathrm{ng} / \mathrm{ml}$.

Statistical analysis. In the present study, all the data were included in the analysis and are expressed as means \pm SD. Statistical significance was calculated using the non-parametric t-test and differences were considered to be statistically significant when $\mathrm{P}<0.05$.

\section{Results}

Clinicopathological characteristics of CRC patients. A total of 240 patients with primary CRC (mean age, $62.0 \pm 12.3$ years; range, 33-89 years), including $117 \mathrm{CC}$ patients (mean age, $62.5 \pm 12.5$ years; range, 33-89 years) and $123 \mathrm{RC}$ patients (mean age, 61.4 \pm 12.1 years; range, $34-86$ years), were recruited for this study. The Duke's stage and other relevant information are summarized in Table I.

Lower CEA level in stage C of CRC differs from that in other stages. The mean value of CEA in patients with CRC in stage A, B, C and D were 34.14, 33.66, 23.35 and $60.05 \mathrm{ng} / \mathrm{ml}$, respectively. Of note, the CEA level in patients with $\mathrm{CRC}$ at stage $\mathrm{C}$ was lower compared to that in any of the other stages, with significant differences from stages A and D (Fig. 1), indicating the significance of stage $\mathrm{C}$, which contrasts the previous hypotheses that CEA levels increased with advancing stage $(12,13)$. Additionally, the CEA level of the non-distant metastasis (NDM) group was different from that in the distant metastasis (DM) group, whereas no differences were observed between the non-metastasis (NM) and metastasis (M) groups. An analysis of other factors, such as patient gender and age, revealed no statistically significant differences (Table II).

CC and RC are generally considered to be of the same nature due to their neighbouring anatomical position. Therefore, the indices of $\mathrm{CC}$ and $\mathrm{RC}$ are analyzed considering these
Table I. Clinicopathological characteristics in colon and rectal cancer patients.

\begin{tabular}{|c|c|c|c|}
\hline & $\begin{array}{c}\text { Total } \\
(n=261)^{*}\end{array}$ & $\begin{array}{c}\text { Colon } \\
(n=129)^{*}\end{array}$ & $\begin{array}{l}\text { Rectum } \\
(\mathrm{n}=132)^{*}\end{array}$ \\
\hline Characteristics & No. $(\%)$ & No. $(\%)$ & No. $(\%)$ \\
\hline \multicolumn{4}{|l|}{ Gender } \\
\hline Male & $139(57.9)$ & $68(58.1)$ & $71(57.7)$ \\
\hline Female & $101(42.1)$ & 49 (41.9) & $52(42.3)$ \\
\hline \multicolumn{4}{|l|}{ Age, years } \\
\hline$\leq 50$ & 45 (18.8) & $19(16.2)$ & $26(21.1)$ \\
\hline$>50$ & $195(81.2)$ & 98 (83.8) & $97(78.9)$ \\
\hline \multicolumn{4}{|l|}{ Duke's stage } \\
\hline A & $57(23.8)$ & $29(24.8)$ & $28(22.8)$ \\
\hline $\mathrm{B}$ & $58(24.2)$ & $31(26.5)$ & $27(21.9)$ \\
\hline $\mathrm{C}$ & $60(25.0)$ & $29(24.8)$ & $31(25.2)$ \\
\hline $\mathrm{D}$ & $65(26.1)$ & $28(23.9)$ & $37(30.1)$ \\
\hline
\end{tabular}

*The sum of all the patients. The data of patients that were outliers is not mentioned in this Table.

Table II. Differences in the serum carcinoembryonic antigen level among patients with colorectal cancer.

\begin{tabular}{lcc}
\hline Variables & Mean, ng/ml & P-value \\
\hline Duke's stage & & \\
A/B & $34.14 / 33.66$ & 0.3508 \\
A/D & $34.14 / 60.05$ & 0.3967 \\
B/C & $33.66 / 23.35$ & 0.2577 \\
B/D & $33.66 / 60.05$ & 0.0711 \\
Gender & & \\
Male/female & $29.47 / 49.70$ & 0.9735 \\
Age, years & & 0.0509 \\
s50/>50 & $10.90 / 43.95$ & \\
\hline
\end{tabular}

two types of cancer as a singe entity. In order to investigate the hypothesis that $\mathrm{CC}$ and $\mathrm{RC}$ may be of different natures, possibly reflected by the differences in the serum CEA level among different stages of CRC (Fig. 1), we divided the cases into two groups and analyzed them accordingly.

Significant differences in serum CEA level are observed among the four stages of $C C$, but not $R C$. The mean values of CEA in patients with CC of stage A, B, C and D were 21.25, $12.37,4.96$ and $113.40 \mathrm{ng} / \mathrm{ml}$, respectively (Table III), whereas those in RC patients were 47.04, 56.28, 42.29 and $15.34 \mathrm{ng} / \mathrm{ml}$, respectively (Table IV). The statistical analysis revealed that $\mathrm{CC}$ exhibited a more significant variation among the four stages (Fig. 2) compared to that in the RC group (Fig. 3). No differences were observed between male and female patients and between patients aged $<50$ and $>50$ years regarding the distribution of serum CEA levels. 
Table III. Differences in the serum carcinoembryonic antigen level among patients with colon cancer.

\begin{tabular}{lcc}
\hline Variables & Mean, ng/ml & P-value \\
\hline Duke's stage & & \\
A/B & $21.25 / 12.37$ & 0.0634 \\
A/D & $21.25 / 113.40$ & 0.0506 \\
B/C & $12.37 / 4.96$ & 0.1792 \\
Gender & & \\
Male/female & $34.16 / 40.26$ & 0.6475 \\
Age, years & & \\
$\leq 50 />50$ & $9.731 / 42.02$ & 0.3259 \\
\hline
\end{tabular}

Table IV. Differences in the serum carcinoembryonic antigen level among patients with rectal cancer.

\begin{tabular}{lcc}
\hline Variables & Mean, ng/ml & P-value \\
\hline Duke's stage & & \\
A/B & $47.04 / 56.28$ & 0.6420 \\
A/C & $47.04 / 42.29$ & 0.7360 \\
A/D & $47.04 / 15.34$ & 0.6140 \\
B/C & $56.28 / 42.29$ & 0.5951 \\
B/D & $56.28 / 15.34$ & 0.3007 \\
C/D & $42.29 / 15.34$ & 0.8738 \\
AB/CD & $51.80 / 28.05$ & 0.3801 \\
ABC/D & $48.50 / 15.34$ & 0.4766 \\
Gender & & \\
Male/female & $24.97 / 59.13$ & 0.6279 \\
Age, years & & 0.0771 \\
S50/>50 & $11.85 / 45.92$ & \\
\hline
\end{tabular}

Serum CEA level is the lowest in stage $C$ of $C C$. Similar to the tendency reported for $\mathrm{CRC}$, the $\mathrm{CEA}$ value in stage $\mathrm{C}$ of CC $(4.96 \mathrm{ng} / \mathrm{ml})$ was lower compared to that in stages A $(21.25 \mathrm{ng} / \mathrm{ml})$ and $\mathrm{D}(113.40 \mathrm{ng} / \mathrm{ml})$. Of note, the CEA level was the lowest in stage $C$ with a significant difference from stages $\mathrm{A}(\mathrm{P}=0.0031)$ and $\mathrm{D}(\mathrm{P}<0.0001)$, as shown in Fig. $2 \mathrm{~A}$. A significant difference was also observed between stages $B$ and $\mathrm{D}(\mathrm{P}=0.0004$; Fig. 2B), whereas no significant difference was observed between stages $\mathrm{B}$ and $\mathrm{C}(\mathrm{P}=0.3216)$. Additionally, due to the high CEA level in stage $\mathrm{D}$ coexisting with the lower CEA level in stages A, B and C, a significant difference was also observed between the NDM and DM groups $(\mathrm{P}<0.0001$; Fig. 2D). However, no difference was observed between the NM and $\mathrm{M}$ groups ( $\mathrm{P}=0.5781$; Fig. $2 \mathrm{C})$. There were no differences among other factors, such as patient gender and age (Table III).

Significant differences in the serum CEA level are observed in stages $C$ and $D$ between $C C$ and $R C$. As described above, the different variation tendencies of the serum CEA level between $\mathrm{CC}$ and $\mathrm{RC}$ prompted the comparison of the CEA level in the
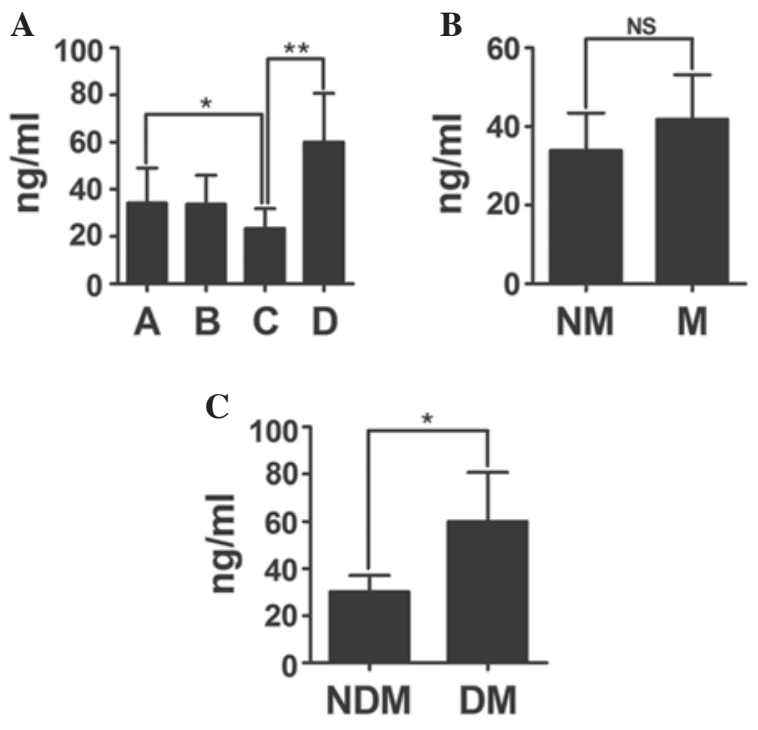

Figure 1. Differences in the serum carcinoembryonic antigen (CEA) level among different stages of colorectal cancer. (A) The CEA level of stage C was found to be lower compared to that in any other stage and exhibited significant variation compared to stages A and D. (B) No differences were observed between the non-metastasis (NM) and metastasis (M) groups. (C) Differences in the CEA level between the non-distant metastasis (NDM) and distant metastasis (DM) groups. ${ }^{*} \mathrm{P}<0.05$ and ${ }^{* *} \mathrm{P}<0.01$. NS, not statistically significant.
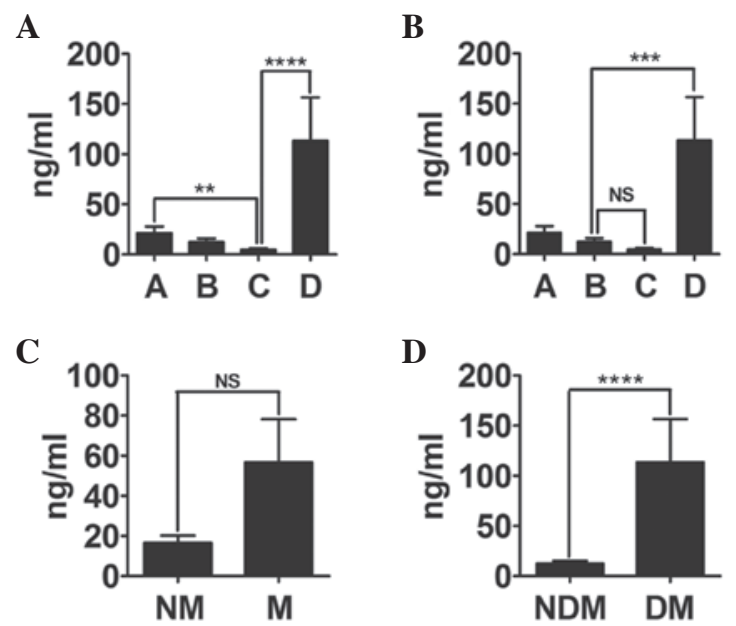

Figure 2. Differences in the serum carcinoembryonic antigen (CEA) level among different stages of colon cancer. (A) The CEA level in stage $\mathrm{C}$ was the lowest among all stages, with a significant difference from stages A and D. (B) The CEA level in stage B was the second lowest among all stages, with differences from stage D. (C) No differences were observed between the non-metastasis (NM) and the metastasis (M) groups. (D) A significant difference was observed between the non-distant metastasis (NDM) and distant metastasis (DM) groups. ${ }^{* *} \mathrm{P}<0.05,{ }^{* * *} \mathrm{P}<0.001$ and ${ }^{* * * * *} \mathrm{P}<0.0001$. NS, not statistically significant.

four stages between $\mathrm{CC}$ and $\mathrm{RC}$. The results revealed no differences in the CEA levels in stage A $(\mathrm{P}=0.9764)$, or in stage $\mathrm{B}$ $(\mathrm{P}=0.1221)$ between $\mathrm{CC}$ and RC (Table V). However, the CEA level in stage $\mathrm{C}$ of $\mathrm{CC}$ was lower compared to that in stage $\mathrm{C}$ of $\mathrm{RC}$, with a significant difference $(\mathrm{P}=0.0096)$; the difference between stage $\mathrm{D}$ of $\mathrm{CC}$ and $\mathrm{RC}$ was also significant $(\mathrm{P}=0.0063)$ (Fig. 4). There were also differences in the CEA level in the NDM group between CC and RC $(\mathrm{P}=0.0155)$, whereas there were no differences in the other factors shown in Table $\mathrm{V}$. 
Table V. Differences in the serum carcinoembryonic antigen level between patients with colon and rectal cancer.

\begin{tabular}{llcl}
\hline & \multicolumn{2}{c}{ Mean $(\mathrm{ng} / \mathrm{ml})$} & \\
\cline { 2 - 3 } Variables & Colon & Rectum & P-value \\
\hline Duke's stage & & & \\
A & 21.25 & 47.04 & 0.9764 \\
B & 12.37 & 56.28 & 0.1221 \\
AB & 16.53 & 51.80 & 0.2465 \\
CD & 56.68 & 28.05 & 0.8897 \\
ABC & 12.52 & 48.50 & $\mathbf{0 . 0 1 5 5}$ \\
ABCD & 36.76 & 39.21 & 0.3516 \\
Gender & & & \\
Male & 34.16 & 24.97 & 0.7250 \\
Female & 40.26 & 59.13 & 0.3373 \\
Age, years & & & \\
S50 & 9.73 & 11.85 & 1.0000 \\
$>50$ & 42.02 & 45.92 & 0.2963 \\
\hline
\end{tabular}

Bold print denotes statistical significance.

\section{Discussion}

Previous studies by Midiri et al (13) and Chen et al (12) reported a different tendency of the serum CEA levels in CRC patients with advancing stage. Our results of the present study revealed that the lowest CEA level was observed in stage $\mathrm{C}$, with significant differences from stages $\mathrm{A}$ and $\mathrm{D}$. The impacts of patient number, age and gender was not taken into consideration as they could not be controlled in this analysis and there were no differences between them. The physical condition of the patients, including the coexistence of other benign diseases (14), may affect the CEA level to a certain extent. Additionally, the $\mathrm{CC} / \mathrm{RC}$ ratio, which was reported to be $56 / 44 \%$ by Chen et al (12) and $58.4 / 41.6 \%$ by Lin et al (15), was different from the $49 / 51 \%$ that was observed in the present study, which may lead to a different tendency if there is internal variation between $\mathrm{CC}$ and $\mathrm{RC}$.

As regards $\mathrm{CC}$, there was significant variation among stages, with the lowest CEA level in stage $\mathrm{C}$, whereas there was no such variation in $\mathrm{RC}$. In addition, $\mathrm{CC}$ and $\mathrm{RC}$ are distinguished by the CEA level in stages $B(P=0.0327), C(P=0.0092)$ and $D$ $(\mathrm{P}=0.0073)$. We cannot exclude the possibility of a deviation arising due to insufficient patient number ( $<30$ in several stages), patient physical condition and statistical error. To reduce the error arising from the insufficient number of patients, several patients were added to the follow-up study; however, the inner differences between $\mathrm{CC}$ and $\mathrm{RC}$ were not affected.

Several studies focused on the hypothesis that $\mathrm{CC}$ and $\mathrm{RC}$ are likely two naturally different diseases; however, the majority of the currently available studies consider CC and RC as a single entity. Wakai et al (2) reported that the dietary risk factors appeared to differ between CC and RC. Li et al (16) reported that the survival of $\mathrm{CC}$ patients was significantly superior to that of RC patients. Nielsen et al (17) also reported
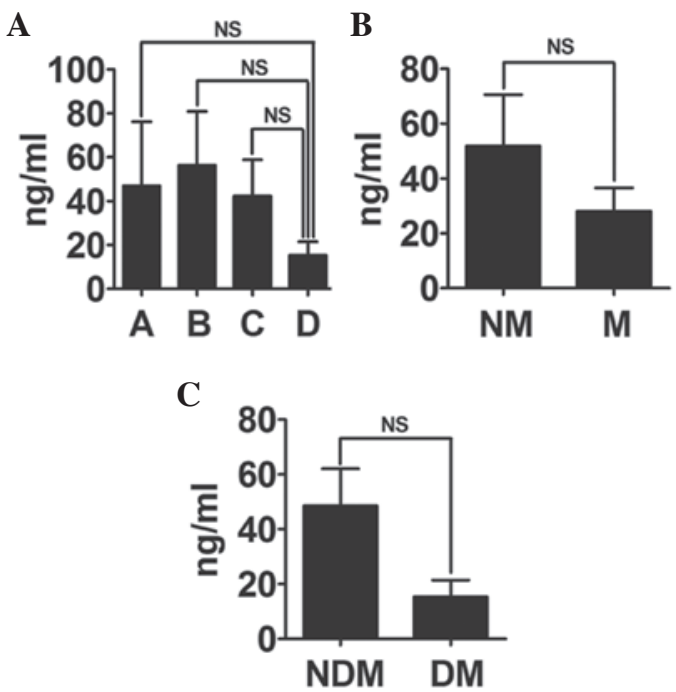

Figure 3. Differences in the serum carcinoembryonic antigen level among different stages in patients with rectal cancer. (A) No significant differences were observed among the four stages of colon cancer. (B) No significant differences were observed between non-metastasis (NM) and metastasis (M) groups. (C) There was no difference between the non-distant metastasis (NDM) and distant metastasis (DM) groups. NS, not statistically significant.

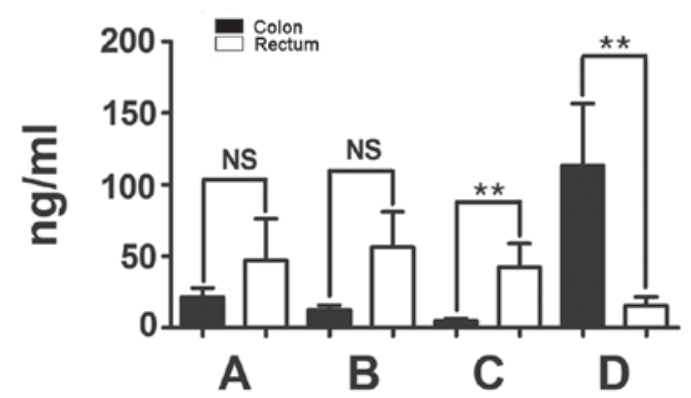

Figure 4. Differences in the serum carcinoembryonic antigen (CEA) level in each stage between colon and rectal cancer. There were significant differences in the CEA level in stages $\mathrm{C}$ and $\mathrm{D}$ between colon and rectal cancer. However, no significant differences were observed in stages A and B. ${ }^{* * *} \mathrm{P}<0.01$. NS, not statistically significant.

that a combination of plasma tissue inhibitor of metalloproteinase 1 and CEA may be useful for the early detection of CRC, particularly CC. Additionally, the Cancer Genome Atlas Network has reported certain differences between tumors from the right colon and all other sites (3). This prompted us to investigate whether the differences in the CEA levels between $\mathrm{CC}$ and $\mathrm{RC}$, despite the overall close association between CRC and CEA, reflect the fact that $\mathrm{CC}$ and $\mathrm{RC}$ are of a different nature, which requires further investigation.

The lowest CEA level was observed in stage $\mathrm{C}$ of $\mathrm{CC}$, with significant differences from stages A and D. Despite the fact that we added more patients in our subsequent study, we cannot exclude the possibility that the patient number was insufficient for analysis, resulting in the unusually low-level of CEA in stage $\mathrm{C}$, which is inconsistent with the findings of Lee et al (18), who investigated 233 stage $\mathrm{C}$ patients and reported that stage $\mathrm{C}$ patients are more significantly associated with increased preoperative CEA levels, as well as the findings of other studies. We also cannot exclude the possibility that there may be a distinction 
between stage $\mathrm{C}$ and other stages in several studies stressing the individual effects of treatment on stage $\mathrm{C}$. Li et al (16) reported that the survival of stage $\mathrm{C}$ patients was significantly superior to that of other CRC patients. Stage $\mathrm{C}$ refers to those patients suffering from cancer cell metastasis to the lymph nodes. The 5 -year overall survival improved as the number of nodes sampled increased for patients with CC (19) and the lymph node ratio is known to be an independent prognostic factor in $\mathrm{CC}$ patients with stage $C$ disease (20). Therefore, the improved survival rate mentioned above for $\mathrm{CC}$ patients with stage $\mathrm{C}$ disease may be correlated with the number of lymph nodes and should be investigated in the follow-up study. Due to the limited number of studies on stage $\mathrm{C}$ of $\mathrm{CC}$, the unique character of stage $\mathrm{C}$ possibly plays a significant role in the evolution of $\mathrm{CC}$; however, the underlying mechanisms require further investigation in the follow-up study.

In the present study, we demonstrated that there is variation in CEA levels among different stages in patients with $\mathrm{CC}$, while no such variation was observed among RC patients. Of note, the CEA level in stage $\mathrm{C}$ was the lowest among all stages in $\mathrm{CC}$ patients. Apart from the factors affecting the results, including age, gender, patient number and physical condition, any unknown internal distinctions between $\mathrm{CC}$ and $\mathrm{RC}$ and between stage $\mathrm{C}$ and other $\mathrm{CC}$ stages remain to be further investigated. Elucidating whether there actually is a difference in the nature of CC and RC may have important implications regarding treatment and prognosis of these two types of cancer.

\section{Acknowledgements}

This study was supported by grants from the Chinese National Science Foundation projects (no. 81372669), the Chinese State Key Program in Basic Research (no. 2012CB822103), the Science and Technology Planning Project of Liao Ning (no. 2012225020) and the Project of Chinese Ministry of Health (no. W2012RQ23).

\section{References}

1. Jemal A, Bray F, Center MM, Ferlay J, Ward E and Forman D: Global cancer statistics. CA Cancer J Clin 61: 69-90, 2011.

2. Wakai K, Hirose K, Matsuo K, Ito H, Kuriki K, Suzuki T, Kato T, Hirai T, Kanemitsu Y and Tajima K: Dietary risk factors for colon and rectal cancers: a comparative case-control study. J Epidemiol 16: 125-135, 2006.

3. Cancer Genome Atlas Network: Comprehensive molecular characterization of human colon and rectal cancer. Nature 487: 330-337, 2012.

4. Mayer RJ, Garnick MB, Steele GD Jr and Zamcheck N: Carcinoembryonic antigen (CEA) as a monitor of chemotherapy in disseminated colorectal cancer. Cancer 42 (Suppl 3): 1428-1433, 1978.
5. Petrioli R, Licchetta A, Roviello G, et al: CEA and CA19.9 as early predictors of progression in advanced/metastatic colorectal cancer patients receiving oxaliplatin-based chemotherapy and bevacizumab. Cancer Invest 30: 65-71, 2012.

6. Gold P and Freedman SO: Specific carcinoembryonic antigens of the human digestive system. J Exp Med 122: 467-481, 1965.

7. Shuster J, Thomson DM, Fuks A and Gold P: Immunologic approaches to diagnosis of malignancy. Prog Exp Tumor Res 25: 89-139, 1980

8. Shively JE and Beatty JD: CEA-related antigens: molecular biology and clinical significance. Crit Rev Oncol Hematol 2: 355-399, 1985.

9. Hefta LJ, Neumaier M and Shively JE: Kinetic and affinity constants of epitope specific anti-carcinoembryonic antigen (CEA) monoclonal antibodies for CEA and engineered CEA domain constructs. Immunotechnology 4: 49-57, 1998.

10. Kojima T, Yoshikawa K, Matsui T, Kodera Y and Kojima H: Titration of serum CEA, p53 antibodies and CEA-IgM complexes in patients with colorectal cancer. Mol Med Rep 2: 477-480, 2009.

11. Edge SB and Compton CC: The American Joint Committee on Cancer: the 7th edition of the AJCC cancer staging manual and the future of TNM. Ann Surg Oncol 17: 1471-1474, 2010.

12. Chen CC, Yang SH, Lin JK, Lin TC, Chen WS, Jiang JK, Wang HS and Chang SC: Is it reasonable to add preoperative serum level of CEA and CA19-9 to staging for colorectal cancer? J Surg Res 124: 169-174, 2005.

13. Midiri G, Amanti C, Consorti F, Benedetti M, Del Buono S, Di Tondo U, Castagna G, Peronace L and Di Paola M: Usefulness of preoperative CEA levels in the assessment of colorectal cancer patient stage. J Surg Oncol 22: 257-260, 1983.

14. Pasanen PA, Eskelinen M, Partanen K, Pikkarainen P, Penttila I and Alhava E: Clinical value of serum tumour markers CEA, CA 50 and CA 242 in the distinction between malignant versus benign diseases causing jaundice and cholestasis; results from a prospective study. Anticancer Res 12: 1687-1693, 1992.

15. Lin JK, Lin CC, Yang SH, Wang HS, Jiang JK, Lan YT, Lin TC, Li AF, Chen WS and Chang SC: Early postoperative CEA level is a better prognostic indicator than is preoperative CEA level in predicting prognosis of patients with curable colorectal cancer. Int J Colorectal Dis 26: 1135-1141, 2011.

16. Li M, Li JY, Zhao AL and Gu J: Colorectal cancer or colon and rectal cancer? Clinicopathological comparison between colonic and rectal carcinomas. Oncology 73: 52-57, 2007.

17. Nielsen HJ, Brunner N, Jorgensen LN, et al: Plasma TIMP-1 and CEA in detection of primary colorectal cancer: a prospective, population based study of 4509 high-risk individuals. Scand J Gastroenterol 46: 60-69, 2011.

18. Lee WS, Baek JH, Kim KK and Park YH: The prognostic significant of percentage drop in serum CEA post curative resection for colon cancer. Surg Oncol 21: 45-51, 2012.

19. Hashiguchi Y, Hase K, Ueno H, Mochizuki H, Kajiwara Y, Ichikura $\mathrm{T}$ and Yamamoto J: Prognostic significance of the number of lymph nodes examined in colon cancer surgery: clinical application beyond simple measurement. Ann Surg 251: 872-881, 2010

20. Elias E, Mukherji D, Faraj W, Khalife M, Dimassi H, Eloubeidi M, Hattoum H, Abou-Alfa GK, Saleh A and Shamseddine A: Lymph-node ratio is an independent prognostic factor in patients with stage III colorectal cancer: a retrospective study from the Middle East. World J Surg Oncol 10: 63, 2012. 